\title{
Analysis of donor-derived cell-free DNA with 3 year outcomes in heart transplant recipients
}

\author{
M. Crespo-Leiro ${ }^{1}$, D. Hiller ${ }^{2}$, R. Woodward ${ }^{2}$, M. Grskovic ${ }^{2}$, C. Marchis2, M. Song ${ }^{2}$, J. \\ Collins $^{2}$, A. Zuckermann ${ }^{3}$ \\ ${ }^{I}$ Hospital Universitario de A Coruña, La Coruña, Spain $;{ }^{2}$ CareDx, Brisbane, CA; ${ }^{3}$ Medical University of Vienna, Vienna, \\ Austria
}

Purpose: Elevated donor-derived cell-free DNA (dd-cfDNA) has been observed in transplant patients undergoing acute rejection, but the relationship between dd-cfDNA and long term outcomes has not previously been studied. In this exploratory study, serially measured dd.cfDNA from heart transplant patients is used for predict 3-year patient adverse outcomes of death, retransplantation, hemodynamic compromise or graft dysfunction.

Methods: cfDNA was extracted from 166 plasma samples representing 48 patients from the CARGO II observational study and dd-cfDNA was quantified using a targeted NGS assay. For each patient, the median and maximum of serial dd-cfDNA scores were calculated. The summarized scores for patients with long-term adverse outcomes and patients without adverse outcomes were compared via two-sample testing and AUC-ROC analysis. Within-patient variability of dd-cfDNA scores for patients who had at least 4 scores was also computed (17 patients total).

Results: Eight of the 48 patients (40 samples) had a adverse outcome and serial dd-cfDNA measurements. Patients were $49 \pm 13$ years old, $79 \%$ male and $96 \%$ caucasian, with no statistically significant difference between groups. Within-patient median dd-cfDNA was significantly correlated with outcome $(\mathrm{p}=0.02$, AUC $77 \%$, 95\% CI 59\%-92\%) but within-patient maximum was not ( $\mathrm{p}=0.11$, AUC 68\%). Analysis using all ddcfDNA results from every patient was also not significantly correlated with outcomes, $\mathrm{p}=0.09$. The AUC for predicting long term outcomes in the CARGO II dataset using variability of gene expression profiling scores was $72 \%$, dd-cfDNA variability was noticeably increased in four patients who an adverse outcome and had four serial AlloSure scores when compared to 13 patients who did not have an adverse outcome.

Conclusion: Median dd-cfDNA score is correlated to long-term outcome. This result suggests that dd-cfDNA merits further investigation as a prognostic marker of long-term risk in heart transplant patients. 